\title{
Consensus Guidelines on Opening Up of Aesthetic Practices in India During the COVID-19 Era
}

This article was published in the following Dove Press journal:

Clinical, Cosmetic and Investigational Dermatology

\author{
Nitin Sethi $\mathbb{D}^{\prime}$ \\ Sukhbir Singh ${ }^{2}$ \\ Jasleen Kaur (iD ${ }^{3}$ \\ Sonia Raghukumar ${ }^{4}$ \\ Chetna Ramchandani ${ }^{5}$ \\ Shuba Dharmana ${ }^{6}$ \\ Karishma Balani ${ }^{7}$ \\ Hema Jain ${ }^{8}$ \\ Meenaz Khoja ${ }^{9}$ \\ Seema Singhal ${ }^{10}$
}

'Plastic \& Cosmetic Surgery, Fortis Hospital, Ludhiana, Punjab I4I00I, India;

${ }^{2}$ Resplendent the Cosmetic Studio, New

Delhi II0048, India; ${ }^{3}$ Department of

Dermatology, SGRDIMS \& R, Amritsar,

India; ${ }^{4}$ Hair Fair Skin Clinic, Kochi, India;

${ }^{5}$ VLCC Clinics, Mumbai, India; ${ }^{6}$ LeJeune

Group of Medspas, Bengaluru,

Hyderabad, India; ${ }^{7}$ SYNOVACARE

Clinics, Mumbai, India; ${ }^{8}$ Health and

Harmony Clinic, Pune, India; ${ }^{9}$ Dermadent

Skin and Dental Clinic, Pune, India; ${ }^{10}$ SPS

Hospital, Ludhiana, India
Correspondence: Nitin Sethi

Plastic \& Cosmetic Surgery, Fortis

Hospital, Ludhiana, Punjab I4I00I, India

Email drnitinsethi@gmail.com
Introduction: The COVID-19 is a pandemic which has affected most people directly or indirectly. It being a communicable disease, the best way to control the disease is to prevent its spread. Lockdown in India has resulted in aesthetic practices all over the country being shut down indefinitely. As things return to normal, most aesthetic practitioners are looking forward to opening practices. Hence, there was a definite need for a consensus on how to safely open up practices in India and at the same time reassure patients coming into these clinics that their safety is paramount.

Methods: This consensus guidelines use the PICO model in its structure. Five critical areas for opening up clinical establishments were identified and approached independently. A questionnaire was prepared using the modified Likert scale, and all the stakeholders were asked to answer the same. Any differences were then resolved with discussion among the stakeholders. The entire study was divided into five subgroups which were then analyzed in detail.

Results: Key recommendations and consensus guidelines were made after detailed analysis. Handy flow diagram reviews of these key areas have been provided. A thorough review of literature was also done on each of the critical areas and recommendations incorporated wherever feasible.

Conclusion: These recommendations have considered the difficulties and cultural issues faced by aesthetic practices in India. They are user friendly, easy to understand and implement. These guidelines would help in reassuring practices to open up safely and continue serving patients with utmost care.

Level of Evidence: Level V, Consensus guidelines.

Keywords: consensus, India, COVID-19, aesthetic clinics

\section{Introduction}

The Novel Coronavirus (COVID-19) was first reported at the end of 2019. Globally, as of May 23, 2020, there have been 5,103,006 confirmed cases of COVID-19, including 333,401 deaths, reported to the WHO. ${ }^{1}$

The pandemic has also affected India with cases being reported from various parts of the country. To curb the menace of the disease, the country was put in lockdown with all non- essential services including aesthetic practices all over the country suspended. However, with the gradual return of normalcy in the country amid the presence of the fear of this infective disease, aesthetic practices all over the country are gearing up to open their services.

The aesthetic sector, unfortunately, is unorganized in our country as there are no defined guidelines to set up practices. Various authors have published numerous 
global consensus papers and practice-based guidelines on how an aesthetic practice should reopen up in these unique circumstances. Most consensus papers lack the presence of the right selection of stakeholders who are part of the paper writing process, which invariably is an essential criterion for publishing consensus guidelines. ${ }^{2}$

Hence, the authors agreed to write guidelines specific to the Indian scenario keeping in mind the right selection of stakeholders and also making sure people practising in different zones were included in this work.

\section{Methodology}

This study was designed keeping in mind the Indian scenario and the common issues faced by aesthetic practices in the country. The research question was defined, and a scoping search of available literature was done on all the major databases like PubMed, Medline and google scholar. A guideline development group was identified, which consisted of plastic surgeons, dermatologists, aesthetic physicians, A microbiologist and an Intensivist with an anaesthesia background who is involved with managing COVID-19 patients.

The research methodology was developed based on the PICO framework. For comparison in the research, it was decided to carry out the suggested guidelines in at least two clinics which were then evaluated by independent reviewers for the feedback to eliminate bias and to validate the proposed guidelines. This also facilitated in achieving the desired outcome.

A core group was identified among the framework development group, and the group identified five different areas of interest under different subheadings. A questionnaire was then developed by the core group using the validated modified Likert scale to establish a consensus among the practice development group. ${ }^{3}$ Each question was later discussed among the different stakeholders, and any differences in opinion were resolved with an open discussion. The decision of the senior authors was considered final in case of a deadlock.

The entire study was divided into five subgroups, each dealing with different aspects of the patient- clinic-doctor experience. The subgroups identified are listed separately in appendix 1. Each subset was extensively researched, and a conclusion derived in the form a flow chart. All recommendations made were keeping in mind the Indian scenario and all the zones present in the country. After the final assimilation of all the data, the recommendations derived were studied as a pilot project at two different clinics in two different parts of the country, and these were then independently assessed by two of the authors (NS, SS) to eliminate bias. All good clinical practice guidelines along with the Declaration of Helsinki were followed for this pilot study including obtaining necessary consents from all the participants.

\section{Results of the Consensus \\ Patient Scheduling}

The COVID-19 pandemic has brought the use of technology to the fore. The recent telemedicine guidelines issued by the ministry of health and family welfare, Government of India, have validated the use of Telemedicine in clinical practice for diagnosis, treatment and prevention of disease and injuries, research and evaluation, and for the continuing education of health care providers. ${ }^{4}$ Making good use of this facility, emphasis must be laid on scheduling clinic visits in advance, and prior appointments must be made compulsory. As much as possible, walk-in patients should be discouraged. Patients must also be instructed at the time of scheduling, that no accompanying persons will be allowed unless the patient requires a caregiver or the patient is a minor.

Well-spaced appointment slots must be designated bearing in mind that sufficient time is allotted not only for the consultation/procedure but also for carrying out the decontamination protocol before engaging the next patient. The consensus group agreed that spacing of appointments by at least 15 minutes would be ideal. If the clinic has more than one doctor consulting on the same day, then different time slots must be used for different doctors.

\section{Risk Stratification}

Considering that even asymptomatic contacts have shown to be potential transmission vectors, it is prudent that every patient should be treated as possibly COVID-19 positive and that all clinical decisions are adapted to this perceived risk. $^{5,6}$ Implementing an algorithm for procedural risk reduction and a pre-visit questionnaire is of paramount importance. We propose a representative survey in appen dix 2. It is vital that this exercise is performed 24-48 hours before the appointment by phone, e-mail or WhatsApp and documented in the patient's chart. It is sensible to schedule appointments only after stratification of the patients into high risk and low-risk groups based on the questionnaire. The patient flow algorithm following this distinction is elucidated in appendix 3. 


\section{Consent}

It has been shown that the surgical/clinical outcome may be affected by a COVID-19 infection during the perioperative period. ${ }^{7,8}$ These unprecedented times call for additional measures, and every procedure must be coupled with a COVID-19-specific consent form and patient declaration form (appendix 4). Similar consent forms are available by various international societies for reference like the American society of Plastic surgeons. ${ }^{2,5}$

In the event of a patient declining to sign a COVID -19 specific consent, thorough contemplation led to the unanimous conclusion that the treatment must be refused. However, on further deliberation, this was noted to be an impractical choice, and it was agreed upon that if the patient is clinically asymptomatic, a compromise may be affected. Patients may be posted for the procedure at the end of the scheduled list, such that the procedure room may be disinfected immediately after. The onus lies with the treating physician whether to accept such patients or not.

\section{Cleaning/Decontamination of Clinics}

It is paramount for all the clinics to enforce thorough decontamination protocols (disinfection and sterilization) because of the ongoing pandemic to ensure the safety of the staff and the patients visiting the clinic.

Coronavirus can remain infectious on inanimate surfaces for up to 9 days. Surface disinfection with $1 \%$ sodium hypochlorite or $70 \%$ ethanol significantly reduces infectivity on surfaces with 1 min exposure time. ${ }^{9}$

Decontamination of clinics can be broadly divided into:

1. Disinfection

a. General disinfection

b. Surface disinfection

2. Sterilization

a. Instruments

b. Personal protective equipment

c. Laser tips

d. Patient care items

3. Air purification

4. Waste management

\section{Disinfection}

A thorough cleaning of the surfaces before disinfection ensures optimal results.

\section{General Disinfection}

It is recommended to start cleaning from the area of least contamination to the area of high contamination. Various disinfectants can be used against many bacteria and viruses; these include $1 \%$ sodium hypochlorite (at a free chlorine concentration of $1000 \mathrm{ppm}$ and $5000 \mathrm{ppm}$ ), $70 \%$ ethyl alcohol, and povidone-iodine ( $1 \%$ iodine).$^{10}$ Use of $1 \%$ sodium hypochlorite for mopping the floors twice a day is an effective method of reducing the microbial load. The evidence related to the type of mop to be used is very scanty and is left to the physician/clinic policy.

Sodium hypochlorite should be freshly prepared every day, and it should not be used on metal surfaces as it is corrosive. ${ }^{11}$

Bathrooms \& toilets, including the faucets, soap dispenser, doorknobs etc. should be cleaned twice a day. First with detergent and then with hypochlorite solution wearing appropriate PPE and the toilet should be flushed while the seats are covered by the lid to prevent any aerosolization. ${ }^{12}$ Holding the flush handle with a tissue while cleaning the toilets is also recommended.

$70 \%$ ethyl alcohol is a powerful disinfectant and can be used to wipe down surfaces where sodium hypochlorite is not suitable like metal surfaces, painted surfaces and fabrics.

\section{Surface Disinfection Guidelines}

Sanitizers should be kept at the entrance of the clinic, reception counter and at high-risk contact surfaces to sanitize hands (preferably foot operated). Before the patient enters the clinic, they should be given shoe covers, a disposable apron, surgical mask, cap, and an environment-friendly bag to keep their belongings which will help in fomite reduction. ${ }^{13}$

The clinic should remove all the decorative items, brochures, magazines from the reception, consulting and treatment rooms. There should be no extra seats in the waiting area, and the patient should be directly escorted to the procedure/consulting room.

It is imperative to rearrange procedure rooms to minimize contamination. The furniture should be the bare minimum. It is better to remove all the other lasers, trolleys out of the room. If one cannot remove, cover them with non-porous cloth/plastic sheet and sanitize/wash the sheet.

Multipurpose trolleys should be replaced by dedicated trolleys for a given procedure only. It's better to avoid using a linen sheet or towel to cover the client while lying down for a procedure, instead use a disposable 
sheet to avoid fomite generation. It is advised to use disposable equipment and ensure its proper disposal. Needles and sharps should be incinerated or placed in the sharp box immediately and disposed of in appropriate bins after each patient. All the surfaces to be wiped down with $70 \%$ ethyl alcohol after each patient.

The cost of consumables would have to be borne by the clinic and should not be passed on to the patient as part of good medical practice.

Ultraviolet disinfection for small devices and currency has been postulated as an effective modality if available. However, the effectiveness of these devices in decontaminating personal items in possession of the HCW's is still unproven. It is advised to place the currency in a plastic bag and leave it in the clinic for at least four days or switch to digital transactions.

\section{Sterilization}

In general, the Spaulding classification system is used to sterilize instruments and patient care items. ${ }^{14}$ However, in the Indian case scenario, we use an autoclave or a hot air oven to sterilize surgical instruments. There must be a designated area within the clinic where all sterilization procedures and storage take place. All instruments and linen to be sterilized should be marked with an indicator to confirm adequate sterilization, as is the standard practice. Reusable surgical instruments should be effectively sterilized to avoid infection to the patient, staff and damage to the instrument. It should be first disassembled (if required), if soiled, then mechanical scrubbing, washed (to reduce the microbial burden), dried then sterilized appropriately. All the effluents should be treated with $1 \%$ sodium hypochlorite solution before being discharged. All the reusable personal care equipment's like goggles, and face shields should be cleaned with 70\% alcohol and kept overnight. For the N95 masks, the CDC Recommends "limiting the number of reuses to no more than five uses per device to ensure an adequate safety margin". The cover is to be stored in a paper bag and kept away for five days and reused on the 6th day. ${ }^{15}$ Laser tips and surfaces coming in direct contact with patient's skin should be cleaned with $70 \%$ ethanol in between each procedure. Patient's care items like the stethoscope, BP cuff, tourniquet etc. should be cleaned with $70 \%$ ethanol after each use.

\section{Air Purification}

Adequate ventilation and filtration help in reducing the airborne transmission of SARS-CoV-2. Proper filter installation and maintenance is necessary. Good ventilation helps to dilute the indoor air contaminants. ${ }^{16}$ In most of our clinics, temperature and humidity are controlled by single units and it is recommended that it should be set to vent open mode. To change the direction of flow of air away from the physician doing the procedure, a fan can also be used. ${ }^{16}$ The biggest challenge is with central air conditioning, as the circulation of air in multiple rooms can potentially be responsible for the spread of communicable diseases like COVID-19. Having an exhaust system in high-risk areas like the reception and the procedure room would certainly help. Air purifiers with HEPA (highefficiency particulate air) filters that are available at affordable cost, which circulate air in a $155 \mathrm{sq}$. ft. Room 5 times per hour can be used instead. ${ }^{17,18}$ For procedures that generate aerosol ${ }^{19}$ like laser treatments, microdermabrasion with crystals, etc., Plume evacuation systems with filters that remove particulates up to 0.1 micrometer known as ULPA (ultra-flow particulate air) filter or smoke evacuators or conventional suction machines should be used. A transparent membrane-like polyvinyl cling film can also be used for aerosol-generating laser (Q-switch laser for tattoo removal).

\section{Biomedical Waste Management, Decontamination Areas}

A specific area needs to be identified for donning and doffing, both for the disposable linen that the patients wear and use and the PPE's that the clinic staff and doctors would use. While for the patient, we recommend an area at the reception be identified for donning and doffing. It can be done in a separate area if technically feasible or in the same area making sure the doffing area is thoroughly disinfected and also making sure there is spacing of timings between the first and the subsequent patients. However, for the staff and the doctor, the donning area shall be the changing room and the doffing area will be the procedure room itself to reduce the amount of contamination in the rest of the clinic. In each procedure room, office, waiting area and restrooms, no-touch waste bins should be lined with disposable liners. Soiled PPE should be disposed in a sealed disposable biomedical waste bag in a bin with a lid and disposed off separately labelled COVID Waste. For contaminated linen, machine washing with warm water at $60-90^{\circ} \mathrm{C}$ is suggested, followed by drying. If machine washing is not possible, linens can be soaked in hot water and soap and then drained, later linens to be immersed in $0.05 \%$ chlorine for approximately 30 
minutes. Finally, the laundry should be rinsed with clean water and the linens allowed to dry thoroughly, if possible in sunlight. ${ }^{20}$

The healthcare waste should be segregated, disinfected and disposed of safely. The person who has healthcare waste should wear appropriate PPE (boots, long-sleeved gown, heavy-duty gloves) and perform hand hygiene after removing it. ${ }^{21}$ The healthcare waste must be collected and put in colour-coded waste disposal bags yellow, red, blue, black, as a specific colour code is followed for different

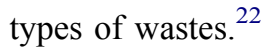

\section{Guidelines for Staff}

In pandemic situations, safety and efficiency of the staff is even more critical for the smooth functioning of the clinic and is the liability of the clinic owner/Doctor. ${ }^{23}$ Hence the specific guidelines related to clinic staff deserve a separate section in this consensus.

The clinic can have rotational duties with shorter working hours for the staff; it is advisable to have $33 \%$ to $50 \%$ of staff working at the clinic at a given point in time. ${ }^{24,25}$

All the clinic staff should be assigned specific areas according to their expertise and should be trained in protocols for physical distancing, hand washing and hand sanitization and surface disinfection. ${ }^{26}$

The staff should be made aware of the WHO guidelines for donning and doffing of PPE, storage and disposal of PPE. ${ }^{27}$

The staff is always at risk of contracting the infection, and this risk should be discussed with the staff before the clinic resumes work. It should be ensured that none of the staff has any signs of COVID-19 before returning work.

A signed consent form explaining the risk of infection to the staff, ${ }^{28}$ and their willingness to still work should be documented. Though staff members may be more comfortable with regular COVID testing for them, it may not be suitable in a limited resource setting like in India. A group insurance cover may be useful for each clinic to cover the expenses of the treatments of the infected staff if they so require. $^{29}$

Older staff ( $>60$ years) or those with comorbidities like respiratory problems, cardiac diseases, diabetes should be given leave or designated work with minimal patient exposure. $^{30}$

The staff should be educated about the signs and symptoms of COVID-19, modes of transmission and the risk factors and high-risk individuals for contracting the disease before the clinic reopens. Not coming to work if they have any symptoms of COVID-19 should also be emphasized upon. The staff should change their street clothes into scrubs or coveralls or clinic uniform and a designated area for changing and storage of the staff belongings should be allotted. Used uniforms should be collected in a laundry bag marked as Biohazard. Every staff must go through a daily temperature check and questionnaire for COVID symptoms. Barriers may be required at the front desk/reception to ensure that patients keep a safe distance from the staff. Barriers may also be needed in the breakrooms or lunch areas for the staff. It should also be ensured that the staff does not gather together for meals and tea breaks. If feasible, outdoor or open spaces should be reserved for clinic staff breaks. ${ }^{29}$ The staff guidelines are elucidated in flowchart form in appendix 5.

\section{Guidelines for Front Desk Staff}

The front desk staff are the most vulnerable to contracting and spreading the disease. Wherever possible, the front desk staff should be encouraged to work through a transparent barrier.

The front desk staff must limit the number of individuals in the clinic waiting area and ensure that physical distancing of 6 feet is implemented. They should also ensure that every patient that enters the clinic, has a cloth or surgical face mask covering the nose and mouth of the patient. ${ }^{29}$

The front desk staff should be made aware of the disposable donning and doffing area for the patient at the reception.

The front desk staff must disinfect areas of frequent contact like the clinic entrance door, reception desk, payment portals, drawer for cash and stationery with sanitizer or $70 \%$ alcohol solution. ${ }^{29}$

It is advisable that an experienced staff manages the phone calls to patients and asks relevant questions to categorize patients into risk categories. The staff can call the patient a day before the appointment and telephonically screen the patients. ${ }^{29,30}$

The staff may also advise the patients on the safety protocol taken by the clinic and the necessary measures that are expected from the patient. The team can encourage the patients to call from near the clinic to check if there are patients in the waiting area so that they can avoid crowding in the waiting area. ${ }^{29}$

The staff should instruct the patients to flush toilets with the lids closed to prevent aerosol formation. ${ }^{12}$ 
The staff should also call the patient after 2-3 days of their visit to check if they have developed any new symptoms related to COVID-19. Any patient who gives positive symptoms should be noted, and all staff in contact with that patient must be screened and preferably quarantined. The staff should be instructed on how to handle product deliveries and vendors. The deliveries may be received outside the clinic entrance to limit the exposure of the patients inside the clinic. Regular vendors should be called at a stipulated time when no patients are scheduled. ${ }^{29}$

\section{Staff Guidelines for Procedures/ Consultation}

Shaving the area for laser treatment or surgical procedures by the patient himself or herself at home should be encouraged by the staff. The patient should be advised to expose only the area which needs treatment or procedure. ${ }^{23}$ The nursing staff in the procedure rooms should wear proper personal protective equipment (PPE) including masks (N95), caps, shoe covers and face shields at all times during the procedure. Sanitization of equipment should also be taught to these staff, and the same should be encouraged in between procedures to prevent cross infections.

\section{Guidelines for Cleaning Staff}

Cleaning staff must wear gowns, surgical masks (N95), shoe covers, gloves and should cover their face with face shield. Nitrile gloves are preferred to latex as they are more chemical resistant; they cause lesser allergic reactions and have higher puncture resistance. ${ }^{31}$ They should be educated about hand hygiene, frequent cleaning of high-touch surfaces with $1 \%$ sodium hypochlorite solution or $70 \%$ alcohol-based sanitizers. Cleaning schedules should be formulated with frequency and name of the cleaner. ${ }^{32}$ There are studies which show that RNA of the SARS-CoV-2 virus is detected in the blood samples and anal swabs of patients. ${ }^{33}$ Therefore, frequent disinfecting of the bathrooms and toilets is essential.

Despite all the above precautionary measures taken, if in the unfortunate event that staff does show symptoms and tests positive for COVID-19, the staff should be quarantined for 14 days. The staff can resume work after five days of the symptoms subsiding. If a staff member tests positive for COVID-19, all the patients in the last two weeks who may have come in contact with the staff must be notified without revealing the identity of the staff member. ${ }^{29}$

\section{Measures for the Doctors Safety Measures and Risk Reduction}

Doctors are encouraged to incorporate teleconsultations where possible, to minimize exposure of oneself and patients to the clinic environment. ${ }^{34}$

Doctors at high risk for Covid-19, ie those over the age of 60 , with diabetes, hypertension, history of coronary artery disease and immune-deficient status are advised to avoid practice where possible. ${ }^{35}$ When not possible, they are encouraged to wear the highest level of protection and to operate with care.

Supplements like Vitamin D has been shown to have some protective effect against viral respiratory diseases in addition to its overall immune conferring benefits. Considering the long confinement the virus has brought along, it is likely that most of the population will be vitamin D deficient now than ever. Daily or weekly dosing has proven to be more protective than monthly dosing. ${ }^{36}$

Other supplements for immune strengthening such as Curcuma, vitamin $\mathrm{C}$ and Zinc can be used through the direct protective effect of these supplements on COVID-19 is unknown and further trials are needed to evaluate their effectiveness. ${ }^{36}$

The Indian Council of medical research has recommended the use of Hydroxy-chloroquine. Trials are still underway to determine its efficacy and actual benefits. However, over the counter use of the drug should be avoided and health care workers are encouraged to use this drug in consultation with their physicians.

PPE with N95 mask, face shield and double gloves is a must for the treating physician more so in cases when performing procedures close to face or aerosol-generating procedures. ${ }^{36}$

Hand washing by doctors is recommended pre and post-procedure after examining a patient or while entering or exiting the clinic. Hand sanitization is a good alternative; however, its efficacy compared to handwashing is considered inferior. Also, the physicians are encouraged to use the right quantity of sanitization solution and for adequate time for disinfection. The amount of sanitizer recommended is 6 to $9 \mathrm{~mL}$ for a contact time of at least 30 seconds. $^{36}$

The donning/disposing of the PPE should be carried out in such a way as to prevent contamination of the HCW's clothes and hands. Gloves should be removed 
first. Gown, shoe cover and head cap must be removed next followed by facemask, glasses. The base layer of gloves should be removed at the end and hands then disinfected by washing or using a sanitizer. The mask should be removed by holding the ear laces and not touching the outside of the mask. ${ }^{37}$ All these things should be disposed of in separate sealed red bag marked as COVID waste. It is recommended to allow as much time as possible for patients to reduce air contamination. The doctor must also leave room only when the procedure is done and re-enter only for the next patient to minimize infection risk. ${ }^{37}$ The protocols for doctors during aesthetic procedures are mentioned in flowchart form in appendix 6.

\section{Doctor Gear}

The masks are of various types. Three-ply surgical masks are more effective at filtering virus particles but not effective against airborne infections. The respirator mask which protects against airborne infection and aerosol-generating procedures $^{36}$ is superior to three ply masks. Respirator masks can be categorised into surgical masks, FFP1, FFP2 (or N95) and FFP3 with $<80 \%, 80 \%, 94 \%$ and $99 \%$ filtration efficacy respectively. Limitation of masks could be overcome by the following suggestions issued by the $\mathrm{CDC}$ guidelines: $(\mathrm{CDC})^{21}$

- N-95 masks can be used up to 8 hours a day and can be combined with a face shield to avoid heavy contamination.

- Minimally soiled N 95 masks can be reused up to 5 times by rotating 4-5 masks every 72 hrs. Each mask, in this case, should be stored separately in a clean bag.

\section{UVC}

UVC light source with a peak wavelength of $254 \mathrm{~nm}$ is a suitable method with a documented germicidal effect on N95 FFRs. A dose of $\geq 1.0 \mathrm{j} / \mathrm{cm} 2$ of UV-C inactivates viruses similar to SARS-CoV-2 ( $\geq 3$-log reduction) on N95 FFRs. The use of UVC irradiation is to be implemented only if there is a severe shortage of N95 FFR. FFR fit may be compromised due to extended cycles of doffing and re- donning.

There are some primary risks associated with UVC, such as direct UV-C exposure is harmful to humans apart from others. Hence UVC is to be included in a risk management protocol and not as complete decontamination. ${ }^{38}$

\section{Procedures}

If performing injectables, doctors, in addition to following the Aseptic no-touch technique protocols, must use double gloves and consider sealing injection points with suitable dressing. ${ }^{21,39}$

The area that is to be injected should be thoroughly cleansed and disinfected with either alcohol, chlorhexidine or iodine and after removing makeup and jewellery. Rinsing the mouth with an antiseptic chlorhexidine mouth wash is suggested as good practice to minimize contamination before the procedure. Preparing a more substantial area around the site of injection for disinfection is highly recommended because of the way the cannula rests against the face distant to the area of injection. Change needles and cannulas between multiple puncture sites. Create an aseptic field on a tray for placing sterile gauze, skin prepping solution and cannulas. ${ }^{40,41}$

\section{During and After Procedure Recommended Guidelines}

The precautions to be taken during procedure begin with Surgical site disinfection protocols which are part of standard operating guidelines. It is imperative to maintain negative pressure inside operating rooms to minimize infection risk. ${ }^{42,43}$ It is recommended to maintain high air exchange cycle rate $\left(\geq 25\right.$ cycles/ $h$ ). ${ }^{44}$ If not possible then, High-Efficiency Particulate Air (HEPA) respirators/ filters can be used which are at least $99.97 \%$ efficient for removing particles $>0.3$ um in diameter. Equipment should be kept minimum about the case only. Surgical trolleys should be prepared beforehand with all the required stuff - staff movement to be kept minimum to avoid crosscontamination and infection. Surgeon, assistants, scrub nurse and anaesthetists should all wear PPE, double gloves, N95 masks, face shield or goggles (protects the conjunctiva). ${ }^{45}$ The patient should be made to wear a mask or FFP filter if available. If the nasal or perioral area needs to be operated, act even more cautiously. If aerosol spread is suspected, follow guidelines as in the flowchart. ${ }^{46-49}$ Perioral and nasal areas to be cleaned thoroughly with betadine as it has superior viricidal effects compared to chlorhexidine. The standard preparation of the operative field should be done with betadine solution as before. Only addition being that disposable drapes should be used whenever available. Needlestick injury should be avoided at any cost. 
At the end of the procedure, separate containers should be prepared for disposing of all sharps; infected instruments; PPE; all the linen if used; shoe-cover and head caps. PPE should be carefully removed at the designated place only and placed inside a sealed red plastic bag and marked as COVID Waste. All BMW (Biomedical waste) protocols to be followed at all times. Hands to be sanitized after removing all the protective gear. A minimum of 10 minutes for health care professional (HCP) to relax is recommended. ${ }^{50}$

\section{Conclusion}

As is often said in aesthetic practice, you can become a safe injector but can never be so, and it's the same when it comes to dealing with a highly communicable disease like COVID-19. As life in the country returns to near-normal levels in most areas, people have started venturing out and want to look at their beautiful self again. So aesthetic practices are bound to see an influx of patients as the sentiment returns to normal. It is thus necessary that we prepare ourselves to ensure the safety of our patients and staff. Following an algorithmic approach in this regard, it would take us a long way in keeping our practices safer and cost-effective. The patients can undergo elective aesthetic procedures based on risk categorization. ${ }^{51}$ This consensus focuses on the critical areas of most aesthetic practices, and by segregating each area under subgroups, management would be easier. Any lapses would be easy to detect and fix. A pilot test of these guidelines indicated that having protocols for key areas was necessary for preventing the spread of this highly communicable disease.

\section{Consent}

The necessary informed written consent for the pilot study from the various participants was taken based on the declaration of Helsinki and are available with the authors.

\section{Acknowledgments}

The authors wish to acknowledge the contributions of Dr. Vinay Singhal, MBBS, MD (Anesthesia), Head Department of Critical care Medicine, Fortis Hospital, Ludhiana and Dr. Suman Sethi, MBBS, MD (Medicine), DM (Nephrology) for their key inputs and in helping of the framing of these guidelines.

\section{Disclosure}

Nitin Sethi reports personal fees from Allergan India, outside the submitted work. The authors report no other potential conflicts of interest for this work.

\section{References}

1. Available from: https://covid19.who.int/. Accessed May 23, 2020.

2. Rosenfeld RM, Shiffman RN, Robertson P. Clinical practise guideline development manual. Otolaryngol Head Neck Surg. 2013;148(1): S1-S55.3. doi:10.1177/0194599812467004

3. Available from: https://www.mohfw.gov.in/pdf/Telemedicine/. Accessed March 25, 2020.

4. Rothe C, Schunk M, Sothmann P, et al. Transmission of 2019-nCoV infection from an asymptomatic contact in Germany. Engl $\mathrm{J}$ Med. 2020;382(10):970-971. doi:10.1056/NEJMc2001468

5. Kissler SM, Tedijanto C, Goldstein E, Grad YH, Lipsitch M. Projecting the transmission dynamics of SARS-CoV-2 through the post-pandemic period. Science. 2020;368(6493):860-868. doi:10.1126/science.abb5793

6. Lei S, Jiang F, Su W, et al. Clinical characteristics and outcomes of patients undergoing surgeries during the incubation period of COVID-19 infection. EClinicalMedicine. 2020;5:100331.

7. Aminian A, Safari S, Razeghian-Jahromi A, Ghorbani M, Delaney CP. COVID-19 outbreak and surgical practice: unexpected fatality in perioperative period. Ann Surg. 2020;272(1):e27-e29. doi:10.1097/SLA.03925

8. Steinmann B, Todt D, Pfaender S, Steinmann E. Persistence of coronaviruses on inanimate surfaces and their inactivation with biocidal agents. J Hosp Infect. 2020;104(3):246-251. doi:10.1016/j.jhin.2020.01.022

9. Sattar SA, Springthorpe VS, Karim Y, Loro P. Chemical disinfection of non-porous inanimate surfaces experimentally contaminated with four human pathogenic viruses. Epidemiol Infect. 1989;102 (3):493-505. doi:10.1017/s0950268800030211

10. Available from: https://www.mohfw.gov.in/pdf/ Guidelinesondisinfectionofcommonpublicplacesincludingoffices.pdf/. Accessed May 18, 2020.

11. Liu Y, Ning Z, Chen Y, et al. Aerodynamic characteristics and RNA concentration of SARS-CoV- 2 aerosol in Wuhan hospitals during COVID-19 outbreak. bioRxiv. 2020. doi:10.1101/2020.03.08.982637

12. Chen Y, Pradhan S, Xue S. What are we doing in the dermatology outpatient department amidst the raging of the 2019 novel coronavirus? $\mathrm{J} \mathrm{Am}$ Acad Dermatol. 2020;82(4):1034. doi:10.1016/j.jaad.2020.02.030

13. Spaulding EH. Chemical disinfection of medical and surgical materials. In: Lawrence C, Block SS, editors. Disinfection, Sterilization, and Preservation. Philadelphia: Lea \& Febiger; 1968:517-531.

14. Available from: https://www.cdc.gov/niosh/topics/hcwcontrols/recom mendedguidanceextuse.html/. Accessed April 7, 2020.

15. Qian H, Zheng X. Ventilation control for airborne transmission of human exhaled bio-aerosols in buildings. $J$ Thorac Dis. 2018;10(S9): S2295S2304. doi:10.21037/jtd.2018.01.24

16. Elias B, Bar-Yam Y. Could air filtration reduce COVID-19 severity and spread? Engl Complex Syst Inst. 2020.

17. Available from: https://www.airsystems-inc.com/air-purificationnews/airquality/the-use-of-hepa-filters-is-vital-in-medical-facilities/. Accessed March 7, 2020.

18. Emadi S-N, Abtahi-Naeini B. Coronavirus disease 2019 (COVID-19) and dermatologists: potential biological hazards of laser surgery in epidemic area. Ecotoxicol Environ Saf. 2020;198:110598. doi:10.1016/j. ecoenv.2020.110598

19. World Health Organization. Water, Sanitation, Hygiene, and Waste Management for the COVID-19 Virus: Interim Guidance. 2020.

20. Safe management of wastes from health-care activities. Geneva: World Health Organization;2014.Available from: https://apps.who. int/iris/bitstream/handle/10665/42175/9241545259.pdf.

21. Bio medical waste management rules, DGHS, CPCB. Accessed May 2019. (Second amendment).

22. Jindal A. Dermatological procedures amidst COVID-19: when and how to resume. Dermatol Ther. 2020;e13561. doi:10.1111/dth.13561

23. Kluger DM, Aizenbud Y, Jaffe A, et al. Impact of healthcare worker shift scheduling on workforce preservation during the COVID-19 pandemic. medRxiv. 2020;2020. doi:10.1101/2020.04.15.20061168 
24. Wei W, Zheng D, Lei Y, et al. Radiotherapy workflow and protection procedures during the coronavirus disease 2019 (COVID-19) outbreak: experience of the hubei cancer hospital in Wuhan, China. Radiother Oncol. 2020:S0167-8140(20)30163-8. doi:10.1016/j.radonc.2020.03. 029.

25. Zheng Y, Lai W. Dermatology staff participate in the fight against Covid-19 in China. J Eur Acad Dermatol Venereol. 2020;34(5). doi: $10.1111 /$ jdv. 16390

26. World Health Organization. Infection prevention and control of epidemic- and pandemic-prone acute respiratory infections in health care. World Health Organization; 2014. Available from: https://apps. who.int/iris/handle/10665/112656/. Accessed May 4, 2020.

27. Zou L, Ruan F, Huang M, et al. SARS-CoV-2 viral load in upper respiratory specimens of infected patients. Engl J Med. 2020;382 (12):1177-1179. doi:10.1056/NEJMc2001737

28. LOS. Angeles Task Force Recommendations. 2020.

29. Khurana A, Sharma KA, Bachani S, et al. SFM India oriented guidelines for ultrasound establishments during the COVID 19 pandemic. $J$ Fetal Med. 2020;7(2):95-101. doi:10.1007/s40556-020-00254-7

30. Chadwick R. Comparison of nitrile and latex examination gloves. $\mathrm{Br}$ Dent J. 2004;196(11):685. doi:10.1038/sj.bdj.4811350

31. Ministry of Health S. Environmental Cleaning Guidelines for Healthcare Settings (Summary Document) Summary of Recommendations. June 2013.

32. Zhang W, Du RH, Li B, et al. Molecular and serological investigation of 2019-nCoV infected patients: implication of multiple shedding routes. Emerg Microbes Infect. 2020;9(1):386-389. doi:10.1080/ 22221751.2020.1729071

33. Sehgal VN. Indian Dermatology. Int $J$ Dermatol. 1993;32 (11):838-844. doi:10.1111/j.1365-4362.1993.tb02782.x

34. Zhou F, Yu T, Du R, et al. Clinical course and risk factors for mortality of adult inpatients with COVID-19 in Wuhan, China: a retrospective cohort study. Lancet. 2020;395(10229):1054-1062. doi:10.1016/S0140-6736(20)30566-3

35. Paprottka KKF, Casabona REG. Elective, non-urgent procedures and aesthetic surgery in the wake of SARS - COVID-19: considerations regarding safety, feasibility and impact on clinical management. Aesthet Plast Surg. 2020;1-29.

36. Coccolini F, Perrone G, Chiarugi M. Surgery in COVID-19 patients: operational directives. World J Emerg Surg. 2020;15(1):25 doi:10.1186/s13017-020-00307-2

37. Mouton C, Hirschmann MT, Ollivier M, Seil R, Menetrey J. COVID-19 ESSKA guidelines and recommendations for resuming elective surgery. J Exp Orthop. 2020;7(1):28. doi:10.1186/s40634-020-00248-4

38. N95 Decon research document' technical report for UV-C based N95 reuse risk management', version 2.0. Accessed April 23, 2020.
39. Heydenrych I, Kapoor KM, De Boulle K. A 10-point plan for avoiding hyaluronic acid dermal filler-related complications during facial aesthetic procedures and algorithms for management. Clin Cosmet Investig Dermatol. 2018;11:603-611. doi:10.2147/CCID.S180904

40. Clare S, Rowley S. Implementing the aseptic non-touch technique $\left(\mathrm{ANTT}^{\circledR}\right)$ clinical practice framework for aseptic technique: a pragmatic evaluation using a mixed-methods approach in two London hospitals. J Infect Prev. 2018;19(1):6-15. doi:10.1177/1757177417720996

41. Centres for Disease Control and Prevention. Coronavirus disease 2019 (COVID-19) situation summary. Available from https://www.cdc.gov/cor onavirus/2019-ncov/summary.html/. Accessed March 2020.

42. Ti LK, Ang LS, Foong TW. What we do when a COVID-19 patient needs an operation: operating room preparation and guidance. Can J Anaesth. 2020;6.

43. Wong J, Goh QY, Tan Z, et al. Preparing for a COVID-19 pandemic: a review of operating room outbreak response measures in a large tertiary hospital in Singapore. Can J Anaesth. 2020;6.

44. Wang D, Hu B, Hu C, et al. Clinical characteristics of 138 hospitalized patients with 2019 novel coronavirus-infected pneumonia in Wuhan China. JAMA. 2020;323(11):1061-1069. doi:10.1001/jama.2020.1585

45. Johnson GK, Robinson WS. Human immunodeficiency virus-1 (HIV-1) in the vapours of surgical power instruments. J Med Virol. 1991;33:47-50.

46. Gloster HM, Roenigk RK. Risk of acquiring human papillomavirus from the plume produced by the carbon dioxide laser in the treatment of warts. J Am Acad Dermatol. 1995;32(3):436-441. doi:10.1016/ 0190-9622(95)90065-9

47. Unadkat SN, Andrews PJ, Bertossi D. Recovery of elective facial plastic surgery in the post-coronavirus disease 2019 era: recommendations from the European academy of facial plastic surgery task force. Facial Plast Surg Aesthet Med. 2020;22(4):4. doi:10.1089/ fpsam.2020.0258

48. Zheng L. Recommendations for surgery during the novel coronavirus (COVID- 19) epidemic. Indian J Surg. 2020;11:1-5.

49. Bertossi D. Safety guidelines for nonsurgical facial procedures during COVID-19 outbreak. J Cosmet Dermatol. 2020. doi:10.1111/ jocd. 13530

50. Kapoor KM. COVID-19 pandemic: consensus guidelines for preferred practices in an aesthetic clinic. Dermatol Ther. 2020;16: e13597.

51. Available from: www.plasticsurgery.org/documents/medicalprofessionals/COVID19-Informed-Consent.pdf. Accessed August 15, 2020 .
Clinical, Cosmetic and Investigational Dermatology is an international, peer-reviewed, open access, online journal that focuses on the latest clinical and experimental research in all aspects of skin disease and cosmetic interventions. This journal is indexed on CAS
The manuscript management system is completely online and includes a very quick and fair peer-review system, which is all easy to use. Visit http://www.dovepress.com/testimonials.php to read real quotes from published authors. 